\title{
Sports prehospital-immediate care and spinal injury: not a car crash in sight
}

\author{
Jonathan R Hanson, ${ }^{1}$ Brian Carlin ${ }^{2}$
}

\begin{abstract}
- Additional supplementary files are published online only. To view these files please visit the journal online (http://dx.doi. org/10.1136/bjsports-2012091800).
\end{abstract}

${ }^{1}$ Emergency department, Dr Mackinnon Memorial Hospital, NHS Highland, Isle of Skye,

Scotland

${ }^{2}$ Centre for Emergency Medical Sciences, School of Medicine, University College Dublin,

Dublin, Ireland

\section{Correspondence to}

Dr Jonathan R Hanson,

Dr Mackinnon Memorial

hospital, NHS Highland, Isle of

Skye, Scotland, IV49 9AA;

jonathanhanson@nhs.net

Accepted 6 October 2012

Published Online First

18 October 2012

\begin{abstract}
The prehospital management of serious injury is a key skill required of pitch-side medical staff. Previously, specific training in sports prehospital-immediate care was lacking or not of a comparable standard to other aspects of emergency care. Many principles have been drawn from general prehospital care or in-hospital training courses. This article discusses sports prehospitalimmediate care as a niche of general prehospital care, using spinal injury management as an illustration of the major differences. It highlights the need to develop the sport-specific prehospital evidence base, rather than relying exclusively on considerations relevant to prolonged immobilisation of multiply injured casualties from motor vehicle accidents, falls from height or burns.
\end{abstract}

\section{INTRODUCTION}

Efforts to standardise immediate care and trauma management developed through courses such as advanced trauma life support (ATLS) in the 1980s. Many similar courses now exist for the initial management of acute medical emergencies and prehospital care. This list has expanded exponentially to include the emergency management of obstetrics (Advanced Life Support in Obstetrics) Surgery (Care of the critically ill surgical patient) and Paediatrics (Advanced Paediatric Life support and Paediatric Trauma Advanced Life Support) among others. Such courses are designed to standardise the early management of common emergency situations through adherence to guidelines, particularly with regard to the less experienced members of the team.

Evidence-based medicine means that some guidelines are frequently revised based upon improved knowledge and evidence-for example the International Liaison Committee on Resuscitation cardiopulmonary guidelines. We now have a far greater understanding of the components of life support that truly make a difference to outcome (minimising interruptions in compressions and early defibrillation $^{1}$ )

Sports prehospital-immediate care covers the skills and techniques needed to safely manage a potentially seriously injured or unwell athlete. Although serious injuries which occur in sport will present to hospitals and be managed via similar skills and guidelines, the actual injury profiles and contributory factors such as mechanism, force, training and environment are quite different from those in the injury spectrums which general prehospital care and ATLS encompass, with the obvious exception of motorsport or equestrian sport. Courses designed with motor vehicle collisions, falls from height or burn and blast injuries in mind, are not directly applicable to serious sporting injury profiles. Hence, sports prehospital-immediate care is a niche of general prehospital care and merits its own specific training, guidelines and evidence base. This discussion aims to cover the major differences between the evidence base for sports prehospital-immediate care and general prehospital care and the current evidence base concerning appropriate techniques. One of the largest areas of development and controversy remains in spinal injury management and we will address the major differences and challenges between poly-trauma and sports injury management.

\section{IMMEDIATE CARE NEEDS OF THE PITCH-SIDE RESPONDER \\ Pitch-side responder versus emergency room practitioner}

Although some countries do recognise sport or sports and exercise medicine as a distinct speciality, the vast majority of pitch-side responders are laymen with simple first-aid training, physiotherapists/athletic trainers or family physicians (general practitioners (GP)), none of whom deal with seriously injured casualties on a regular basis. When they do, they are often unsupported, have minimal equipment often in challenging conditions such as poor light or bad weather. They also deal with casualties seen immediately postexercise, who are normally tachypneoic and tachycardiac - but with a picture of improvement. Contrast this to the emergency room practitioner who sees many seriously injured patients and has immediate assistance and equipment to hand as well as specialist support readily available in the same building.

\section{Limitations of in-hospital courses in sport}

The pitch-side responder will be involved in the immediate stabilisation of the injured athlete. This may include packaging the casualty on a spinal device with the assistance of local paramedic support services. The emergency room practitioner will be dealing with the removal of the casualty from the device for further assessment, rather than putting them on the device. Hence, the courses and training relevant to the emergency room practitioner are not designed for the work environment or contain the necessary skills which a GP or physiotherapist needs to manage the potentially critically injured sportsman on the field of play. In-hospital courses such as ATLS do not provide the skills needed for working pitch side. ${ }^{2}$

Similarly, with regard to sudden athletic cardiac death (incidence of around 1 in $43000 /$ year $^{3}$ ) the improved availability of automated external defibrillators suits those who lack skills in analysing 
cardiac rhythm and lack familiarity with intravenous access and drugs. Yet the major in-hospital resuscitation courses such as Advanced Cardiac Life Support (ACLS) or Advanced Life Support cover other areas such as pacing or periarrest arrhythmias, none of which is relevant to the pitch-side medic.

\section{Strengths and weaknesses of general prehospital-care courses in sport}

General prehospital qualifications such as the Pre-Hospital Emergency Care Certificate (PHECC) are designed to improve the local immediate care response to accidents, often by family physicians in rural areas. They also standardise prehospital-care skills among those who frequently respond and work in the prehospital environment. Hence, the target group for this course better reflects the background of the pitch-side responders. However the injury spectrum for which the course is designed reflects relatively high velocity polytrauma such as motor-vehicle accidents with tissue loss and a higher likelihood of requiring invasive intervention. Skills such as endotracheal intubation, or open thoracostomy are of less relevance and inappropriate for pitch-side responders.

Spinal handling and packaging skills are a crucial component of both prehospital-care training and sports-immediate care, but for various reasons provoke controversy and wide regional variation of 'best practice' in terms of techniques and equipment.

\section{SPINAL INJURY IN GENERAL PREHOSPITAL CARE VERSUS SPORTS-IMMEDIATE CARE \\ Preextrication considerations}

Multiple-injured casualties are scored via injury severity scores as a predictor of mortality. Compared with complex polytrauma from high-velocity injuries, the majority of sporting serious injury is of a low severity score. Cervical spine injury is associated with high-injury severity scores in blunt trauma, but in lower-velocity trauma (such as may be seen in sport) the risk of spinal injury is not related to the actual force of collision to the head or neck, but rather to whether the athlete falls or not after the assault. ${ }^{4}$ The typical prehospital-potential spinal injury patient may have the added difficulties of:

1. Multiple injuries

2. High-velocity trauma

3. Complex extrication from entrapment

4. Multiple casualties

5. Hazardous uncontrolled environment such as a motorway.

All of these will influence the speed, choice of equipment and techniques for extrication and spinal immobilisation.

\section{On-field considerations}

With the exception of motor and equestrian sports, injury data from field or team sports rarely show any of the above, although in head injuries it is not uncommon to have two unconscious sporting casualties. The typical hazards of the on-field environment may be limited to weather and ongoing match play, all of which are usually of lower risk and controllable as opposed to risk of explosions or oncoming vehicles.

Hence the factors that influence the choice of equipment and techniques are different in terms of rescuer experience and casualty environment, and so it is important to consider the individual working environment and sport when deciding on the most appropriate equipment and technique.

\section{Extrication and postextrication considerations}

A further area where there is a fundamental difference in spinal management between general prehospital and sports-immediate care is the speed of response of the immediate-care practitioner. In sport it is truly immediate, the mechanism may be directly witnessed and a response will usually be possible within seconds. This is not the case with general prehospital care where there will inevitably be a delay between the incident occurring and an emergency-response vehicle or ambulance arriving. With regard to spinal care and the need for immobilisation, this is relevant to the application of guidelines based upon whether the casualty is unconscious or not on arrival of the rescuer. If a casualty remains unconscious after a few minutes, this is obviously significant, but in fact the majority of sporting loss of consciousness seen by pitch-side responders is much more transient. Some will be deemed to have a Glasgow Coma Scale of 15 on the pitch and skilled staff may be able to 'clear' the cervical spine on-field and walk off rather than need to be immobilised.

Similarly, all casualties from the prehospital environment who undergo spinal immobilisation will remain packaged until arrival at an emergency department. This is not the case with sports immediate care cases, where match day situations with experienced medical support with transient loss of consciousness as the precautionary indication for immobilisation, many can be clinically cleared of serious cervical spine injury and removed from the device at the ground. Hence, considerations such as pressure problems from prolonged immobilisation on rigid spinal devices are less relevant. ${ }^{5}$

\section{EVIDENCE-BASED SPINAL HANDLING TECHNIQUES}

Traditional techniques such as log rolling, semirigid collars and long-spine boards have recently being challenged on the grounds of increasing segmental spinal motion and the risk of complications from local pressure ${ }^{5}$ or raised intracranial pressure from tight fitting collars. 6 Similarly, the evidence on which some of those challenges are being made is being questioned ${ }^{7}$ particularly in terms of cadaveric models. Hauswald describes cadaveric simulation of catastrophic spinal injury as a poor comparison with the in vivo situation due to rigour mortis producing abnormally 'stiff' healthy levels. Also the lack of oedema and swelling from an artificially induced penetrating transection produces an unrealisticically hypermobile injured segment ${ }^{7}$ in contrast to the swelling and oedema within an injured spinal level and enveloping soft tissues producing hypomobility at the injured site. He also argues that traumatic cervical spinal injuries are either minor or catastrophic and of the 1-2\% which sit somewhere in between (all causes), the vast majority are stable injuries where casualty handling during rescue cannot produce a sufficient force to convert them to a catastrophic cord injury. Such forces will never be reproduced during a sporting extrication and the increased stiffness related to swelling and bleeding at the injured level are postulated as a reason for a rethink of the current management of prehospital-spinal care, arguing that the benefits of traditional spinal immobilisation are minimal but the potential complications from equipment are unfavourable. Historical theories about the link between spinal injuries and unconsciousness are also being challenged with head injury failing to be an independent risk factor for spinal injury over suspicious mechanism. ${ }^{8}$

Various techniques for controlling the head and cervical spine while performing spinal packaging are described in the literature.

- Manual inline stabilisation (head squeeze)

- Trap squeeze

- Log roll

- Lift-and-slide technique

- High aim in endangered spine (H.A.in.E.S recovery position) The head squeeze reflects the common manual inline stabilisation or MILS $^{2}$ that is a fundamental part of ATLS and 
prehospital care teaching. It involves simply kneeling or lying behind the casualty and placing a hand over either side of the head to protect the region from movement or accidental contact. The 'trap squeeze" is similar but involves placing the hands either side of the root of the neck and gripping the trapezius so that the head lies between the forearms. A log roll ${ }^{10}$ involves a controlled manoeuvre to keep the cervical spine in line while rolling the casualty onto their side to allow positioning on a spinal device. Rather than rolling, the lift-and-slide ${ }^{10}$ technique involves lifting a supine casualty vertically off the ground a few centimetres and sliding the device underneath them, this avoiding any roll. The high arm in endangered spine manoeuvre involves using an extended arm to support the head during rolling or in a recovery position, thus producing less downward lateral flexion. ${ }^{11}$

The majority of evidence related to spinal handling in sport relates to low numbers and is experimental. The literature suggests that the lift-and-slide technique produces least movement in the cervical spine with lateral flexion and that rotation a particular concern with a head squeeze and log roll combination. However, the lift-and-slide technique is only applicable to supine casualties, and yet anecdotal (You Tube) and injury surveillance reviews suggest that unconscious athletes fall in many positions and so will require rolling. A review by Mobbs, which concludes that the avoidance of the log roll and use of split devices or the lift-and-slide technique is preferable also concedes that prone casualties still need to be log rolled onto a device. ${ }^{12}$ As sporting casualties fall in a host of positions, the log roll remains a key skill in pitch-side immediate care (figure 1).

The majority of traumatic loss of consciousness in sport occurs at low speed and athletes collapse depending on the posture at the time of impact. Contrast this to general prehospital care situations where unconscious casualties may travel through the air at considerable speed after being ejected from a vehicle and will have sufficient momentum to roll and ultimately come to rest subject to the physics of the centre of mass and centre of gravity. Not surprisingly in man, the mass of the spine and posterior musculature result in centres of gravity and mass which lie posteriorly and ejected rolling casualties often end up in the supine position.

Furthermore, the lift-and-slide technique depends on rescuers gripping sports clothing to perform the lift. With modern 'performance' compression clothing often worn in sports the techniques may not be applicable to all sports. Those with large protective equipment may be easier to lift. The H.A.in.E.S. manoeuvre uses an extended arm at the shoulder to buttress the lateral flexion of the head and cervical spine during log

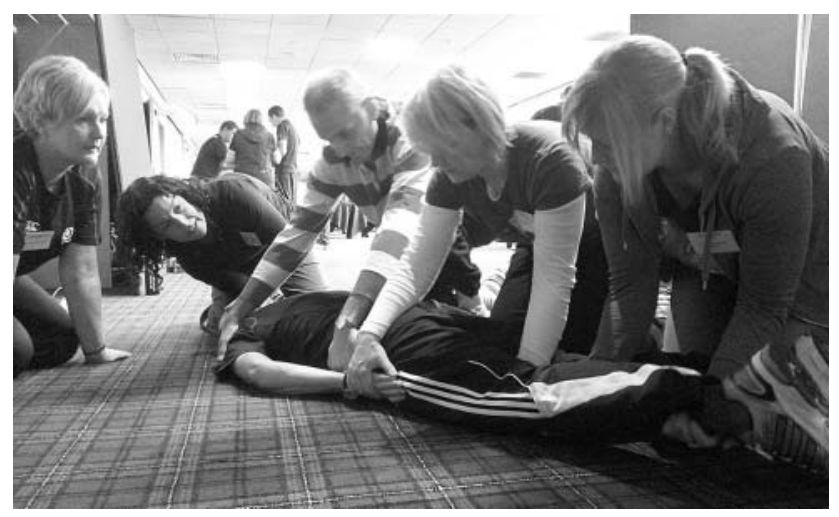

Figure 1 Prone log roll training. rolling. Although logic suggests that the ideal minimum spinal movements during handling are as low as possible there is no evidence for absolute measurements despite studies referring to minimum desired movement. We simply do not know 'how much is too much?'

A further general prehospital care argument against the log roll relates to disruption of haematomas in a fractured pelvis or significant visceral injury. ${ }^{5}$ However pelvic ring disruption would be a very rare injury in most sports and due to the speed of response of the pitch-side practitioner, haematomas would be unlikely to have had time developed during pitch-side handling

In conclusion, the evidence favouring particular spinal handling techniques in sport remains weak and laboratory studies do not address practical challenges such as sports clothing, the common position of casualty at rescue or establish a scientific threshold for clinically relevant safe cervical movement. From a practical perspective it would be elegant to choose techniques which cause as little lateral flexion or rotation as possible relative to the position in which the player is lying. Hence, if supine, avoiding a log roll is preferable and either a lift-and-slide (depending on clothing) or scoop-to-board technique would be preferable. In an emergency such as vomiting, using the H.A.in. E.S manoeuvre ${ }^{11}$ to reduce downward lateral flexion would be preferable to a standard roll.

\section{Clearing the cervical spine on field}

Decisions about whether to spinally immobilise a casualty or whether the immobilisation can be removed in hospital depends upon a series of evidence-based guidelines (Nexus ${ }^{13}$ or Canadian $\mathrm{C}$-spine ${ }^{13}$ guidelines). These are designed around the mechanism of injury (falls from height, expelled from vehicle), the pattern of injury (distracting injuries, injury above the clavicle) and the clinical state of the patient (GCS, neck tenderness and distal neurological symptoms). Only some of the variables considered are likely in sport and often the specifics of the mechanism within sport may go unnoticed. Although the Nexus or Canadian C-spine guidelines are useful, they are only partially relevant to pitch-side work.

\section{Choice of spinal equipment in sport}

Further geographical variation exists as to the optimum spinal extrication device. From a sports perspective the common presentation in which we spinally immobilise a casualty would be a transient loss of consciousness in which we cannot rule out cervical spine injury, although other injury patterns do exist. Classical spinal immobilisation consists of semirigid collar, blocks and straps on a supportive device-the so called 'triple immobilisation'. Although sports providers may practice spinal immobilisation techniques regularly, the likelihood is that they will use their skills infrequently over a season. With retention of practical skills such as cardio-pulmonary resuscitation (CPR) being shown to fall by $86 \%$ at 1 year, ${ }^{13}$ familiarity with equipment, guidelines and practice are a crucial part of performing a skill correctly. Hence, while regular practitioners such as ambulance staff may improvise with devices for triple immobilisation for example, using bags of fluid and sports tape, the infrequent practitioner should use only dedicated equipment.

Semirigid collars are, however, not without their own controversy with studies suggesting that they may offer little protection from excessive intersegmental motion of the cervical spine in stable or unstable vertebral columns. ${ }^{6}$ This is despite various designs and styles. The cadaveric model on which many of these studies are based have, however, been challenged as a poor model. ${ }^{7}$ 


\section{IMMEDIATE CARE PLANNING LARGE VERSUS SMALL POPULATION}

Athletes do not fit the mould of everyday people. Depending on the particular sport, a large proportion of athletes may be taller, wider, heavier or distorted by protective equipment than the statistics for the general public. Although bariatric equipment is available for the morbidly obese, abdominal girth is not usually the challenges of handling large athletes.

In terms of the ergonomics of a device, the emergency medical services use equipment covering the anthropometrics of the general population and cover SDs from the mean in a normal distribution. Pitch-side responders will have different anthropometrics and may deliberately plan devices and techniques to cope with those out with the normal range. Obviously, there needs to be co-operation with the ambulance service over equipment compatibility, but a significant proportion of match day incidents do not leave the stadia and are clinically cleared on site (figure 2 ).

\section{STRENGTHS AND WEAKNESSES OF VARIOUS SPINAL EXTRICATION DEVICES \\ Long spine board}

The traditional spinal immobilisation device is simply a flat board which can have an attached dedicated triple immobilisation system. Most devices cover up to $2 \mathrm{~m}$ and come in various widths and thicknesses. It is versatile and can be used for rapid take downs of standing or sitting casualties as well as prone, supine or irregular casualties. Longer ones are available or have extra width/shapes to accommodate protective padding etc. They are out of favour in some regions due to concerns about pressure issues in those with spinal cord injury and casualties should not remain on them for more than 30 min. ${ }^{5}$ Padded ones are available however.

\section{SPLIT DEVICES}

These include the aluminium orthopaedic scoop stretcher, the Ferno 65 XL (modern scoop) and the 'combi-board'.

Split devices are popular in prehospital circles as they can avoid a log roll in supine casualties or help extricate from a vehicle. ${ }^{14}$ However, it is designed as a transfer device and should not be carried for any distance. Those who are not supine will still need to be rolled. ${ }^{12}$ Triple immobilisation systems are not commonly found on the traditional aluminium or modern polypropylene versions and they need to be improvised. The combiboard has a system with a solitary head strap. Split devices can be narrow (Ferno $65 \mathrm{XL}-43 \mathrm{~cm}$ ) and require improvised cervical spine control. The ergonomics of the device is variable with the

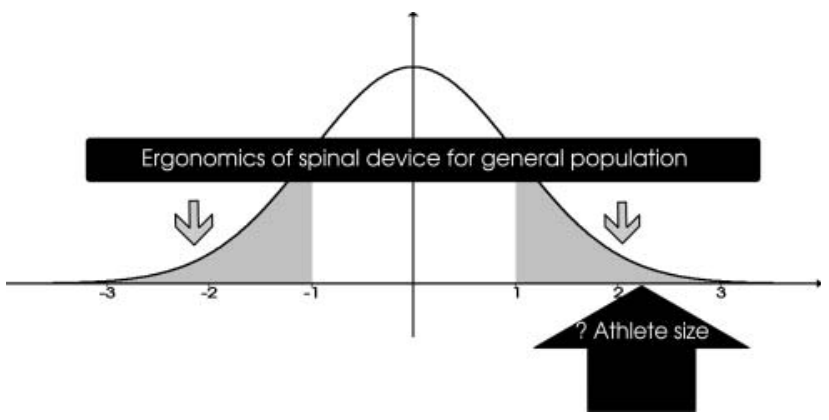

Figure 2 Ergonomic considerations for a spinal device for the general population, versus the potential ergonomics of a distinct athlete group. Pitch-side responders may need to plan differently from the ambulance service. aluminium version being subject to bowing when carrying heavy athletes-particularly when extended to its maximum length $(2 \mathrm{~m})$. Our measurement of the maximum shoulder width of elite rugby forwards showed a range up to $65 \mathrm{~cm}$ and hence any such athlete carried on a narrow split device would be unstable-particularly as the handles are embedded within the $45 \mathrm{~cm}$ of the device rather than additional to it. They are useful for transferring a supine casualty onto another device or buggy but should not be used for carrying heavy athletes (table 1).

\section{VACUUM MATTRESS}

Perhaps the gold standard device in true spinal cord injury, the vacuum mattress provides an individually moulded cocoon for the casualty through a double bagged polystyrene ball system which becomes rigid when the air is removed. They come in a range of sizes and widths and avoid the problems of local pressure areas as the force is evenly spread out along the whole body. However, if they puncture, the valve fails or the pump is lost, then they become of little value. Hence they should always be used with a backup device available. In the UK they are only slightly more expensive than a long spine board and are becoming more and more popular with the ambulance service as the device of choice.

\section{OXYGEN}

Spinal cord injuries remain an important part of sports immediate care. As with general prehospital responders, our management may influence secondary cord injury through movement, bleeding and cord hypoxia. Oxygen therapy has recently become less popular in conditions such as myocardial infarction where the oxidative and free radical effect is thought to have a negative impact on survival in normoxaemic patients. ${ }^{14}$ However, no such evidence exists in spinal care and when faced with an unconscious or potentially spinally injured patient in the first few minutes, when it is unclear as to the full severity or spectrum of injury, oxygen therapy at $15 \mathrm{l} / \mathrm{min}$ is still recommended. Providing oxygen will fill the respiratory dead space so that if there is respiratory or cardiovascular compromise from the head or spinal injury, the rescuer will have more time to act before the casualty becomes critically hypoxic.

\section{SUMMARY}

- Sports prehospital care is a niche of general prehospital care.

- Pitch-side responders use immediate care skills occasionally and so need dedicated equipment.

- Athlete size and shape may influence choice of equipment.

- Complex polytrauma guidelines are less relevant to sport.

- Pitch-side medics respond sooner than ambulance staff and a higher proportion of cases be cleared of serious injury at the ground once removed from the field of play.

Table 1 Professional rugby player shoulder widths - relevant to split device width of $43 \mathrm{~cm}$ with recessed handles

Shoulder and chest width of forwards of a Glasgow Warriors professional Rugby Union team

\begin{tabular}{lll}
\hline Position & Chest width $(\mathbf{c m})$ & Shoulder width $(\mathbf{c m})$ \\
\hline Front row & 47 & 57 \\
Front row & 52 & 62 \\
Front row & 49 & 60 \\
Front row & 44 & 59 \\
\hline
\end{tabular}


Sports prehospital immediate care is a niche of general prehospital care. Differences in the background, experience and working environment of the rescuers, as well as the injury spectrum and speed of response mean that current guidelines, techniques and evidence base are only partly relevant and in some cases irrelevant to providing a pitch-side response. Courses designed for in hospital training and resuscitation (ATLS, ACLS, etc) do not cover the skills need to work pitch side and should not be recommended.

Despite differences in prehospital opinion, the log roll and the (ideally padded) long-spine board remain important techniques for extrication of an athlete with a potential spinal injury and remain preferable to carrying a heavy athlete on a split device without dedicated triple immobilisation. Split devices have their place, but only as transfer devices. Vacuum devices overcome the problems of pressure areas and provide better spinal support in casualties, but carry a very small risk of failure. They provide gold standard immobilisation in spinal injury. There is currently some discussion about the negative impacts of spinal immobilisation, particularly semirigid collars and evidence against sporting head injury as an independent risk factor for spinal injury.

Sports prehospital-immediate care courses do exist and we should use these courses to develop our own evidence base for best practice on a sport by sport basis. The size and shape of the athletes, the environment and pitch-side support available will also vary greatly between sports and choices may reflect this.

Contributors Both authors were involved in the planning and conceptualisation of the article, including the key topics covered and differences between general and prehospital care. The article was initially written by $\mathrm{JH}$, with $\mathrm{BC}$ reviewing and editing as appropriate. Both authors have reviewed the final edition and thus meet criteria 1 , 2 and 3 for authorship via the ICMJE guidelines.
Competing interests None.

Patient consent Obtained.

Provenance and peer review Commissioned; internally peer reviewed.

\section{REFERENCES}

1. Hazinski MF, Nolan JP. 2010 International consensus on cardiopulmonary resuscitation and emergency cardiovascular care science with treatment recommendations. Circulation 2010;122:S250-75.

2. American college of surgeons on trauma. Advanced Trauma Life Support manual. 8th edn. Chicago: American College of Surgeons, 2010.

3. Harman KG, Irfan MA, Klosser D, et al. Incidence of sudden cardiac death in National Collegiate Athletic Association Athletes. Circulation 2011;123:1594-1600.

4. Kulvatunyou N, Friese RS, Joseph BB, et al. Incidence and pattern of cervical spine injury in blunt assault: it is not how they are hit, but how they fall. J Trauma Acute Care Surg 2012;72:271-5.

5. Kwan I, Bunn F. Effects of prehospital spinal immobilization: a systematic review of randomized trials on healthy subjects. Prehosp Disaster Med 2005;20:47-53.

6. Mobbs RJ, Stoodley MA, Fuller J. Effect of cervical hard collar on intracranial pressure after head injury. ANZ J Surg 2002;72:389-91.

7. Hauswald M. A re-conceptualisation of acute spinal care. Emerg Med J 2012;00:1-4.

8. Hasler RM, Exadaktylos AK, Bouamra 0, et al. Epidemiology and predictors of cervical spine injury in adult major trauma patients: a multicenter cohort study. J Trauma Acute Care Surg 2012;72:975-81.

9. Shrier I, Boissy P, Briere S, et al. Can a rescuer or simulated patient accurately assess motion during cervical spine stabilization practice sessions? J Ath/ Train 2012;47:42-51.

10. Del Rossi G, Horodyski M, Powers ME. A comparison of spine-board transfer techniques and the effect of training on performance. J Ath/ Train 2003;38:204-8.

11. Gunn BD, Eizenberg N, Silbertstein MM, et al. How should an unconscious person with a suspected neck injury be positioned? Prehosp Disaster Med 1995;10:239-44.

12. Gutteridge GA, Conrad $B P$, Rossi $G D$, et al. Eliminating log rolling as a spine trauma order. Surg Neurol Int 2012;3(Suppl 3):S188-97.

13. Smith KK, Gilcreast D, Pierce K. Evaluation of staff's retention of ACLS and BLS skills. Resuscitation 2008l;78:59-65.

14. Wijesinghe $\mathbf{M}$, Perrin $\mathrm{K}$, Ranchord A, et al. Routine use of oxygen in the treatment of myocardial infarction: systematic review. Heart 2009;95:198-202. 